\title{
Automatic Target Recognition in Synthetic Aperture Sonar Images Based on Geometrical Feature Extraction
}

\author{
J. Del Rio Vera, ${ }^{1,2}$ E. Coiras, ${ }^{1}$ J. Groen, ${ }^{1}$ and B. Evans ${ }^{1}$ \\ ${ }^{1}$ NATO Undersea Research Centre (NURC), 19126 La Spezia, Italy \\ ${ }^{2}$ ESRIN, European Space Agency (ESA), 00044 Frascati, Italy \\ Correspondence should be addressed to J. Del Rio Vera, jorge.del.rio.vera@esa.int
}

Received 31 July 2008; Revised 2 December 2008; Accepted 3 March 2009

Recommended by Athanasios Rontogiannis

This paper presents a new supervised classification approach for automated target recognition (ATR) in SAS images. The recognition procedure starts with a novel segmentation stage based on the Hilbert transform. A number of geometrical features are then extracted and used to classify observed objects against a previously compiled database of target and non-target features. The proposed approach has been tested on a set of 1528 simulated images created by the NURC SIGMAS sonar model, achieving up to $95 \%$ classification accuracy.

Copyright (c) 2009 J. Del Rio Vera et al. This is an open access article distributed under the Creative Commons Attribution License, which permits unrestricted use, distribution, and reproduction in any medium, provided the original work is properly cited.

\section{Introduction}

Underwater imaging has a wide range of applications, from pipeline inspection to seabed classification and underwater object classification [1]. Although both optical and acoustic sensors can be used for underwater imaging, the working range of the optical sensors is

severely limited (a few meters), even in clear water conditions. This makes acoustic systems the preferred option for underwater imaging, with ranges up to hundreds of meters independently of the water turbidity.

One of the most widely used acoustic systems is the sidescan sonar, which was developed in the 1950s and has evolved along the years [2]. Sidescan sonars can provide highresolution images of the seabed but resolution along the path of the sonar (the azimuth or along-path direction) decreases with range (across-path direction), limiting the effective swath width, and also depends on the aperture (number of wavelengths of the array) of the receiver, with large arrays or very high frequencies being required for higher levels of resolution. For several years, there has been ongoing work on the development of synthetic aperture sonar (SAS) to overcome these limitations. SAS systems eliminate the decrease of resolution with range [3] by stacking more pings at longer range, analogous to the approach used in airborne and satellite radar systems for decades [4]. Recently, SAS has started to become a commercial reality following the solution of the motion estimation problem. SAS systems are now able to provide images of constant resolution, which for recent platforms, such as NURC's MUSCLE vehicle, result in full swath images with resolutions of as good as about 20 $\mathrm{mm} /$ pixel (see Figure 1). This improved image quality makes SAS sensors potentially highly beneficial for underwater automatic target recognition (ATR) tasks.

Broadly speaking, ATR schemas rely on the computation of different types of image features, which can be grouped in three main categories:

(i) texture-based features, which depend on patterns and local variations of the image intensity [5],

(ii) spectral (or radiometric) features, based on spectral characteristics of the backscatter radiation of the targets (i.e., color, energy) [6],

(iii) shape-based (or geometrical) features, which rely on spatial form or contour information extracted by different means (i.e., length, area) [7].

Traditionally, ATR systems based on side-scan have relied on a combination of radiometric and geometric features to identify objects of interest, focusing mainly on the spectral highlight response produced by the target and the configuration of the shadow cast on the seafloor. By 


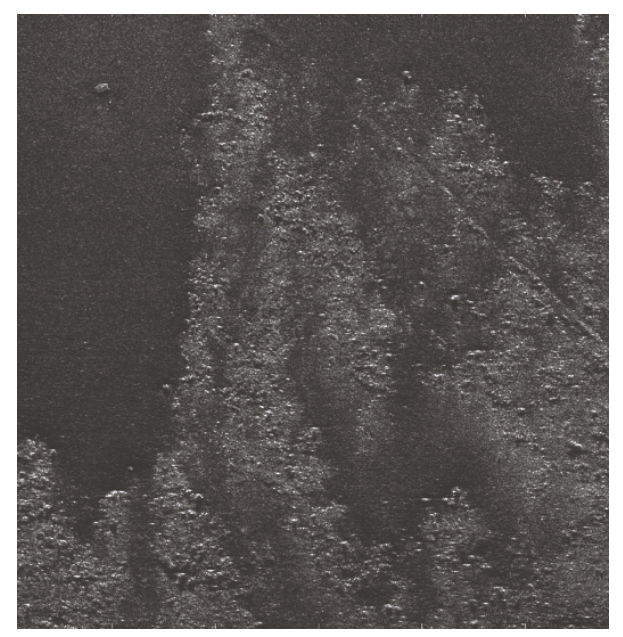

FIGURE 1: High-resolution synthetic aperture sonar image showing the acoustic backscatter from a patch of seabed measured with the MUSCLE system by NURC off the coast of Latvia. Range runs downward and along-track from left to right. The total image is 30 by 30 meters.

using SAS, more detailed information for the shadow and highlights of underwater targets is available, which could be exploited to improve classification results.

In this paper, we present a new supervised classification approach for target recognition in SAS images. The approach uses geometrical features and aims to make use of the increased image fidelity available in both target highlight and shadow response. The recognition procedure starts with a novel detection/segmentation stage based on the Hilbert transform [8], which partitions the image into highlights and shadow areas in order to estimate the most likely position of the target. A number of geometrical features are then extracted around the estimated target position, and are then used to classify the object against a previously compiled database of target and nontarget features.

This paper is organized as follows: Section 2 provides a description of the datasets used for testing and validating the proposed approach; the detection and segmentation scheme are discussed in Section 3. The geometrical features used for classification are explained in Section 4, while the classification schema and the classification results for different parameters are shown in Section 5. The geometric feature extraction methods are applied to a small set of images recorded with the MUSCLE system in Section 6. Finally conclusions are drawn in Section 7.

\section{Data}

Development and evaluation of an automatic target recognition (ATR) system requires an appropriate test data set. Given that high-resolution SAS data is scarce and ground truth knowledge is often lacking, we have used NURC's simulator (SIGMAS) Synthetic Image Generator for Modeling Active Sonar to generate the data used for this work. SIGMAS allows the generation of SAS and side-scan test sets for arbitrary

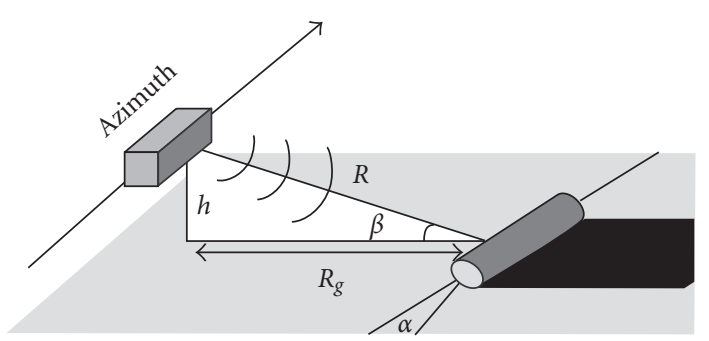

FIgURE 2: Sonar acquisition geometry. The sensor is imaging a cylinder at a range $\mathrm{R}$.

target models, including bottom topography effects such as ripples, sea bottom slope variations, and partial burial of targets [9].

Figure 2 shows the general sonar acquisition geometry. The sensor is flying at a height $h$ over the sea bottom, imaging a rget sitting on the seabed. The target is at a range (distance) $\mathrm{R}$ and is seen under a grazing angle $\beta$ from the sensor. Aspect angle $\alpha$ specifies the orientation of the target with respect to the azimuth direction. The ground range to the target is $R_{g}$, the result of projecting the distance $\mathrm{R}$ on the seafloor. The area of the seabed shown in black is being shadowed by the target, and is, therefore, not scattering any energy back to the sensor.

The images are simulated in several steps.

(i) First, the background level is computed for each pixel assuming Lambertian scattering off the sea floor, which results in lower values for pixels corresponding to shallow grazing angles.

(ii) Subsequently, shadow regions in the image are identified by ray-tracing, which results in pixels set to zero. Penumbral regions, that is, regions that are shadow only in a part of the synthetic aperture, are accounted for.

(iii) The target response is stacked to this template, which is accomplished through ray-tracing under the assumption of a constant sound speed in the water column. The target can have an arbitrary 3D shape, which is decomposed into facets each having their own travel time (or range R) and amplitude. In this way for each facet, the corresponding pixel is determined, whilst the amplitude is computed using a Lambert's scatter law in combination with the Rayleigh reflection coefficient that depends on the angle between facet normal vector and acoustic ray.

(iv) The target response stacking operation is repeated two times in order to include first- and secondorder bottom multipath arrivals of the object. The bounces on the sea floor are affecting the response via spreading and via an amplitude reduction according to [10].

(v) The penultimate step in the process transforms the smooth template into a sonar image by adding the pixel to pixel amplitude variability which is characteristic of the fluctuations in acoustic pressure. For 


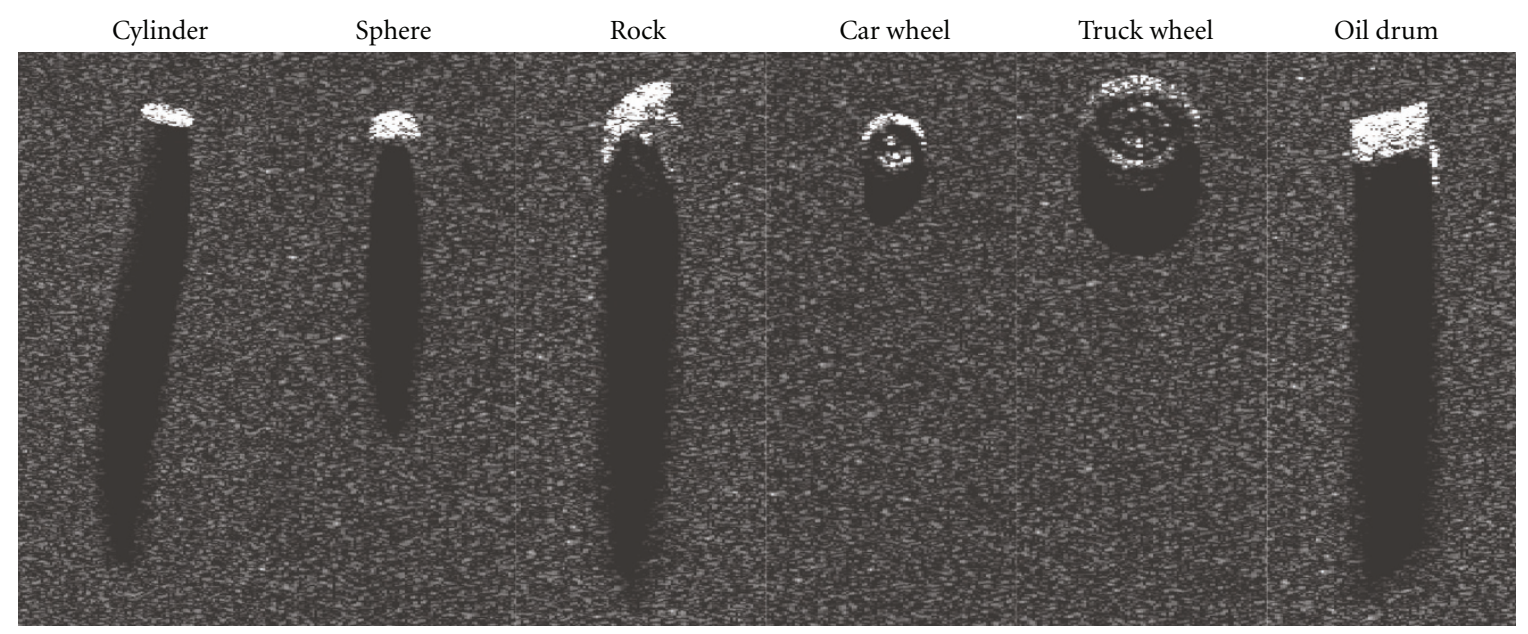

FIgURE 3: Examples of SAS image snippets in the database. The range axis is pointing downward.

this model, the commonly used Rayleigh statistical distribution is used to adjust both highlight and reverberation responses.

(vi) Finally, the image is convolved with the SAS (or sidescan) impulse response to account for sonar resolution and side lobes. This convolution is performed in the 2D Fourier space of the image.

The use of a realistic simulator such as SIGMAS permits to evaluate the sensitivity of the ATR system to various configuration parameters, for example, sonar height or aspect angle of the target. The variations in performance caused by differences in grazing and aspect angles are evaluated in this paper.

The dataset used for the testing described in this paper contains a total of 1528 simulated SAS images comprising six different objects (cylinder, sphere, rock, car wheel, truck wheel, oil drum). The resolution of the images is $25 \mathrm{~mm} / \mathrm{pixel}$ in azimuth and $8.6 \mathrm{~mm} / \mathrm{pixel}$ in range. The sea bottoms slope up and down by up to 2 degrees and the bottom type varies from mud to coarse sand. Target burial depths vary from 0 to $10 \mathrm{~cm}$. Rotation angles for targets in every axis range from -5 to +5 degrees in azimuth and range directions, while the aspect angle varies from -180 to 180 degrees. The sonar height over the seabed ranges from 5 to $40 \mathrm{~m}$ and the targets are placed at ranges between 25 and $200 \mathrm{~m}$ enabling a wide range of sonar to target geometries to be examined. Some examples of the images contained in the database are shown in Figure 3.

Whilst showing only six simulated images, Figure 3 demonstrates many of the fundamental ATR issues and the sensitivity of the final image to small changes in viewing parameters. The difficulty of visually classifying these images indicates that the overall ATR performance is highly dependent on the specific dataset used to test the system. The image on the far left shows an example that contains good discriminative information for a cylindrical target shape. The third image contains much less information and could be from several different shapes, depending on orientation and the amount of burial. Successful classification in this latter case will almost certainly require a second view on the object from a different direction. We can also see that the sea floor characteristics have an important effect on the visual classification, and will show that they have a large influence on classification with geometric features as well.

\section{Detection}

A novel technique based on the Hilbert transform (HT) [8] is used for target detection. The HT can be seen as a border detector even in the presence of noise, is easy and fast to compute [11], and can also be used as the basis of more advanced edge detection techniques, such as the phase congruency technique [12].

It is possible to discriminate between shadow and highlight by using the analytic signal of the image. The analytic signal of $f(t)$ (1D case) is a complex signal $\tilde{f}(t)$ for which $f(t)$ is the real part and $\hat{f}(t)$ is the imaginary part given by the Hilbert transform of $f(t)$ :

$$
\hat{f}(t)=\frac{1}{\pi} P \int_{-\infty}^{\infty} \frac{f(\tau)}{t-\tau} d \tau
$$

where $P \int_{-\infty}^{\infty} f(x)$ is the principal value of the integral.

The amplitude of the analytic signal $\tilde{f}(t)$ provides a clear differentiation of the highlight areas in the image, while its phase can be used to robustly discriminate shadowed regions. For the $1 \mathrm{D}$ case, a target 1 meter wide can be regarded as a step signal $\Pi(t)$, which is 1 if $t \epsilon[-1 / 2,1 / 2]$, and 0 elsewhere. The HT for this shows a constant phase of $\pi / 2$ for the shadowed areas, because the real part of the HT tends to zero as can be verified in the formula derived for a step function:

$$
\hat{\Pi}(t)=\frac{1}{\pi} \ln \left|\frac{t+1 / 2}{t-1 / 2}\right| .
$$

For SAS images, the HT is applied in the range direction line by line, applying the argument expressed for 1D signals. Once shadows and highlights are segmented, the 
potential positions of targets are inferred by assuming they produce a highlight immediately followed by a shadow with dimensions that match that of an object of a given size at the current range from the sensor. Since the training and evaluation images used in the database contain a single target, in what follows only the most likely target location is considered, yet for processing full images the procedure should be applied to all likely locations that are found.

The result of the application of the HT to a simulated image of a car wheel imaged at 100 meters range is shown in Figure 4.

Once the separation in shadow and highlight of the potential target is done, its edge closer to the sonar is extracted by analyzing the highlight. Targets of interest have smooth surfaces and are expected to produce a uniform and strong backscatter signal, as opposed to the noisier and weaker signal returned by the coarser surface of the seafloor. Taking these characteristics into account, a simple algorithm for discarding most of the ripples and elements of topography can be applied: the maximum of every line of constant azimuth is computed, and a curve joining all the maxima is generated; those maximal points are then removed and the process repeated selecting the new maxima from the remaining pixels and generating a new curve. The number of times the procedure is iterated depending on the size of the targets for the given range. For the results presented in this paper, the procedure has been applied, on average, 1/3 times the expected target size in pixels, which amounts to 16 times. The curves resulting from this iterated procedure are shown in Figure 5.

Then, the standard deviation of all the curves is computed for each azimuth value $a$. If it is less than $1 / 3$ of the expected size of the target, then the pixel is considered part of a potential target. The longest curve fulfilling the standard deviation criteria, $C_{h}(a)$, is picked up as the one marking the target's front shape and will later be used by the geometrical features extraction algorithm.

\section{Feature Extraction}

Geometric features are commonly used for classification in controlled environments such as integrated circuit manufacture plants, where the position and orientation of the object with respect to the light source are known [13]. The proposed ATR system for sonar uses the same type of geometric features but in a less controlled environment where the target can present any aspect to the sonar and the signal-to-noise ratio is much smaller. To do so, 24 geometrical features have been selected to differentiate a set of targets. The features are computed from the segments extracted in the detection stage (Section 3). Nine features concentrate on the highlight area, twelve focus on the shadow, and three extract information from the low backscatter area in between them. The low backscatter area is defined as the region that is limited in the range direction by a highlight segment (with pixel values above a given threshold $t_{h}$ ) and a shadow segment (below $t_{s}$, see Sections 4.1, 4.2, and 4.3 for more details).
Details on the highlight and the low-backscatter area contain useful information for discrimination among target types, but have generally not been exploited due to the limited resolution of older sonar sensors, where the highlight typically consisted of just a few pixels.

The geometrical feature extraction starts by approximating the curve fitted to the highlight, $C_{h}(a)$. The subscript $h$ refers to the highlight, whereas $a$ is the azimuth coordinate and $C_{h}$ are the range values defining the curve for each a (Figure 6(b)). Ramer's algorithm [14] is used to approximate $C_{h}(a)$ by a list of linear segments (see Figure 6(a) for a brief demonstration of Ramer's algorithm).

(i) Get the two extremes $C_{h}\left(a_{1}\right)$ and $C_{h}\left(a_{2}\right)$, of the curve (crosses in Figure 6).

(ii) Compute the straight line $\lambda(a)$ from $C_{h}\left(a_{1}\right)$ to $C_{h}\left(a_{2}\right)$.

(iii) Select the corner $C_{1}=\left(a_{c}, C_{h}\left(a_{c}\right)\right)$ that maximizes the distance in range coordinate from $C_{h}(a)$ to $(a) \lambda$.

(iv) Add $C_{1}$ to $\lambda(a)$.

(v) Divide $\lambda(a)$ in two segments $\left[C_{h}\left(a_{1}\right), C_{1}\right]$ and $\left[C_{1}, C_{h}\left(a_{2}\right)\right]$ and recursively apply the algorithm to them.

(vi) The algorithm stops once four corners $\left(C_{1}\right.$ to $\left.C_{4}\right)$ have been found. The use of more corners does not provide better performance for the dataset used.

For each recursion level, three scores are computed:

(i) maximum-range distance from $C_{h}(a)$ to $\lambda(a)$ (score $\left.S_{1}\right)$;

(ii) sum of range distances from $C_{h}(a)$ to $\lambda(a)$ (score $\left.S_{2}\right)$;

(iii) sum of the range distances from $C_{h}(a)$ to the polyline defined by the two segments $\left[C_{h}\left(a_{1}\right), C_{1}\right]$ and $\left[C_{1}, C_{h}\left(a_{2}\right)\right]$ (score $\left.S_{3}\right)$.

Once the approximation by linear segments is done, the following corners are retained to compute features from the highlight: corners $C_{h}\left(a_{1}\right)$ and $C_{h}\left(a_{2}\right)$ and the four corners with the lower $S_{3}$ scores.

4.1. Highlight Features. The distribution of the corners on the highlight segment enables the computation of several parameters to geometrically describe the target, the corners being one of the following features.

(i) Highlight corners: these corners are computed using the algorithm described in Section 3, and then centered to the center of gravity of the highlight segment:

$$
\begin{aligned}
& c g=\left(a_{c g}, r_{c g}\right) \\
& =\left(\frac{\sum_{a} \sum_{r} H(a, r) \cdot a}{\sum_{a} \sum_{r} H(a, r)}, \frac{\sum_{a} \sum_{r} H(a, r) \cdot r}{\sum_{a} \sum_{r} H(a, r)}\right),
\end{aligned}
$$

where $H(a, r)$ is the highlight mask (one if a pixel belongs to the highlight segment, zero elsewhere). 


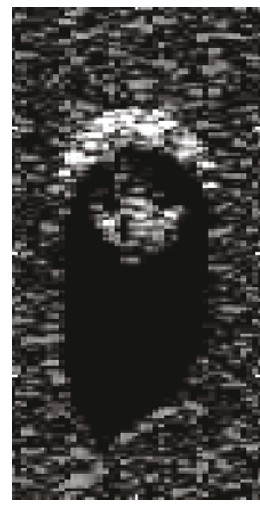

(a)

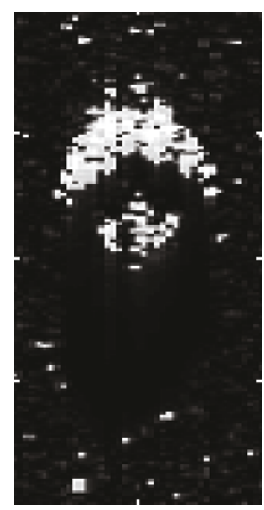

(b)

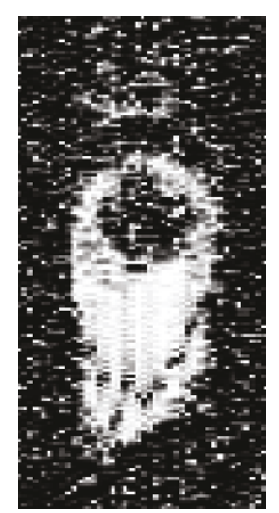

(c)

FIGURE 4: Synthetic SAS image of a car wheel on a sand seabed. (b) Modulus and (c) phase of the Hilbert Transform of (a).

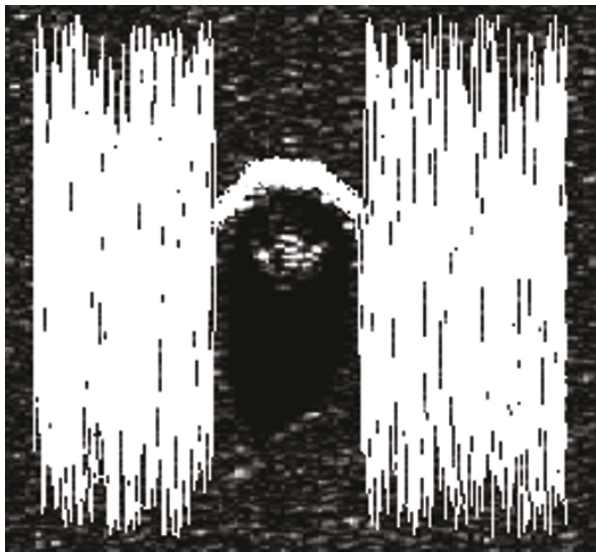

FIGURE 5: Curves obtained by iteratively computing the maximum values for every azimuth (horizontal coordinate in the image). In the target area the variation of these curves is small because the target return is uniform, however, outside the target, since the sea bottom is noisy, the curves exhibit large variations.

(ii) Highlight significant directions: computed by applying principal component analysis (PCA) [15] to the corner distribution. The significant directions are the orientations of the principal components axis. The values used are angles formed by these axes and the azimuth direction.

(iii) Highlight significant directions' scores: the PCA gives a score to the two significant directions it extracts (the normalized value of the eigenvalues). These two scores $P_{1}$ and $P_{2}$ (with $P_{1}>P_{2}$ ) are used as values of significance.

(iv) Length of the longest axis of the highlight $\left(l_{l}\right)$.

(v) Length of the shortest axis of the highlight $\left(l_{s}\right)$.

(vi) Highlight real eccentricity: The real eccentricity is computed using the actual lengths of the target.

$$
E_{1}=\sqrt{1-\frac{\left[l_{s}\right]^{2}}{\left[l_{l}\right]^{2}}} .
$$

(vii) Highlight PCA eccentricity: the two values of significance $\left(P_{1}\right.$ and $\left.P_{2}\right)$, instead of the lengths of the axis, are used to compute eccentricity of the highlight. This reduces the effect of outliers that can affect the length of the axis.

(viii) Ratio of longest to shortest axis.

(ix) Correlation of corner distribution of the highlight with a semicircle: a semicircle of $50 \mathrm{~cm}$ diameter is correlated with the corner distribution to obtain an indicator of target roundness.

4.2. Shadow Features. Corners on the shadow segment are also extracted, although the curve $C_{s}(a)$ for the shadow boundary is computed differently. Only the shadows observed right after the highlight segment are considered. Then azimuth line by azimuth line where there is shadow content, the range value closest to the sonar and belonging to the shadow is taken to create $C_{s}(a)$. Once this curve is obtained, four corners and extremes are computed using the algorithm described in Section 4, and the same nine features are estimated for the shadow segment. Additionally, three extra features are extracted.

(i) Shadow area divided by range.

(ii) Shadow width in contact with highlight.

(iii) Correlation with the shadow-edge model of an object of interest, for instance from a mine.

4.3. Low Backscatter Area Features. The area delimited by the shadow and highlight curves is also used to compute some geometrical features.

(i) Low backscatter highlight's centre of gravity (COGLB).

(ii) Area of low backscatter highlight.

(iii) Distance between the corners of the highlight and the corners of the shadow. 


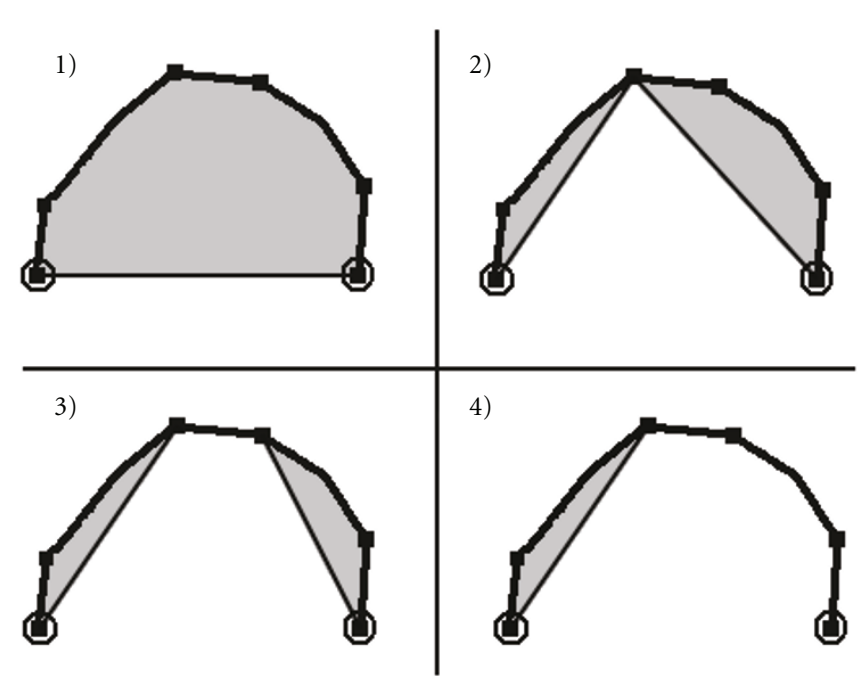

(a)

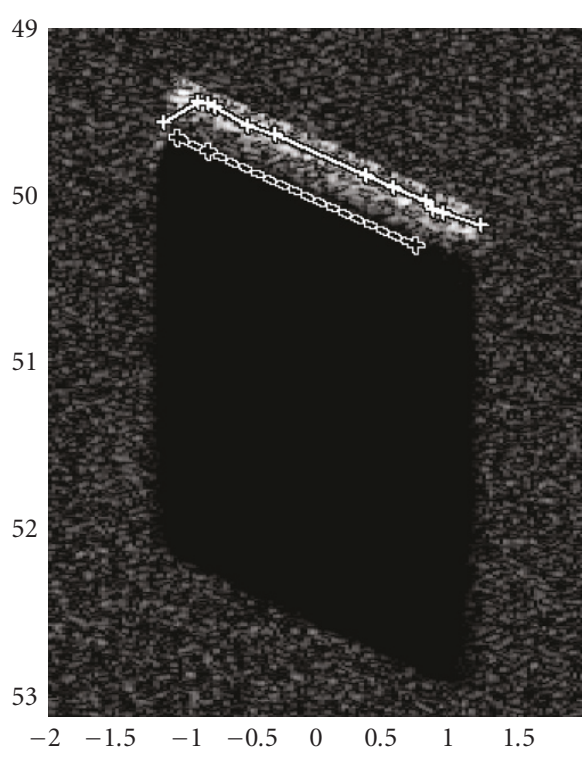

(b)

Figure 6: (a) An example of Ramer's algorithm on a simple curve Step 1) showsthe schematic curve $\left(C_{h}(a)\right)$ which corners shall be found (all corners of the curve are marked with a cross, while only the most relevant ones will be detected (subsequently marked with circles)), the two extremes of the curve $\left[C_{1}, C_{2}\right]$ are marked with circles and the area delimited by the curve can be found as the area inside the area described by the curve and the segment delimited by the two extremes of the curve. Then the algorithm looks for the first corner as the point belonging to the curve $C_{h}\left(a_{1}\right)$ which minimizes the area delimited by $\left[C_{1}, C_{h}\left(a_{1}\right)\right]+\left[C_{h}\left(a_{1}\right), C_{2}\right]$, which is marked with a circle. Then the algorithm is applied recursively to obtain 3$)$ and 4$)$. (b) The $C_{h}(a)$ and $C_{s}(a)$ curves of a real target where the algorithm will be performed. In white the curve $C_{h}(a)$ for the highlight segment from the image of a cylinder (axes in meters). The extremes of the curve $C_{h}\left(a_{1}\right)$ and $C_{h}\left(a_{2}\right)$ are the left-most and right-most white crosses, the rest of the crosses marks all the possible corners of $C_{h}(a)$. Also shown in black is the curve $C_{s}(a)$ that delimits the start of the shadow region.

\section{Classification Results}

For classification we used the MATLAB tree function [16], trained to discriminate a particular type of target against all other types, effectively creating a set of binary classifiers for the targets of interest (one classifier per target class, discriminating between that class and all other classes). This common approach $[17,18]$ gives information about the worst performance of a non binary classifier against the worst target:

$$
\begin{gathered}
\sum_{i \neq j} \mathrm{P}\left(c \notin C_{i}\right) \cdot P\left(C_{i}\right)=P\left(c \notin C_{\bar{j}}\right) \cdot P\left(C_{\bar{j}}\right) \text {, hence } \\
P_{i \neq j}\left(c \notin C_{i}\right) \cdot P\left(C_{i}\right) \leq P\left(c \notin C_{\bar{j}}\right) \cdot P\left(C_{\bar{j}}\right),
\end{gathered}
$$

where $C_{i}$ represent classes and $c$ is an object to be classified. The six classes considered in this study were cylinder, sphere, rock, car wheel, truck wheel, and oil drum.

Classification performance using the proposed geometrical features has been estimated by cross-validation. The dataset (1528 simulated SAS images as described in section 2) was divided into two random halves, one used for training and the other for validation. The process was repeated 100 times to obtain the average performance estimates. Receiver operating characteristic (ROC) curves were then produced for each object class.

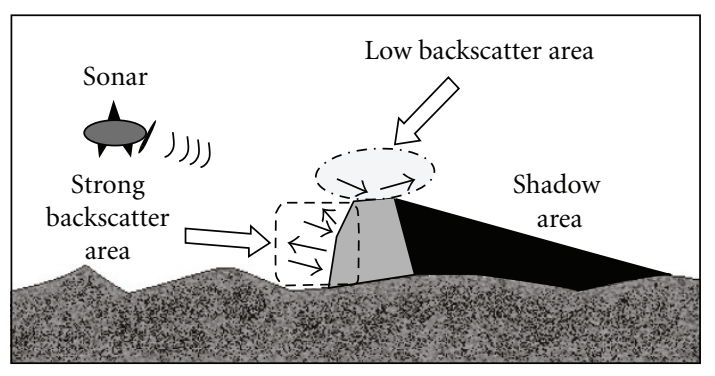

FIgURE 7: Areas which can be distinguished in the sonar image of a proud object on the seabed due to the different levels of backscattered signal.

Figure 8 shows the ROC curves obtained for four classes in the database, including the best and the worst cases found (cylinder and oil drum, resp.).

Results show the best performance for cylinders, well described by the lengths of the longest and shortest axis (longest axis of $2.4 \mathrm{~m}$ with the following longest axis being the truck wheel at $1.2 \mathrm{~m}$ ). Nevertheless, targets of similar sizes, such as the sphere and the wheel, were still well differentiated. The worst case was found to be the oil drum, which for certain aspect angles and burial depths is confused with several other target classes. 


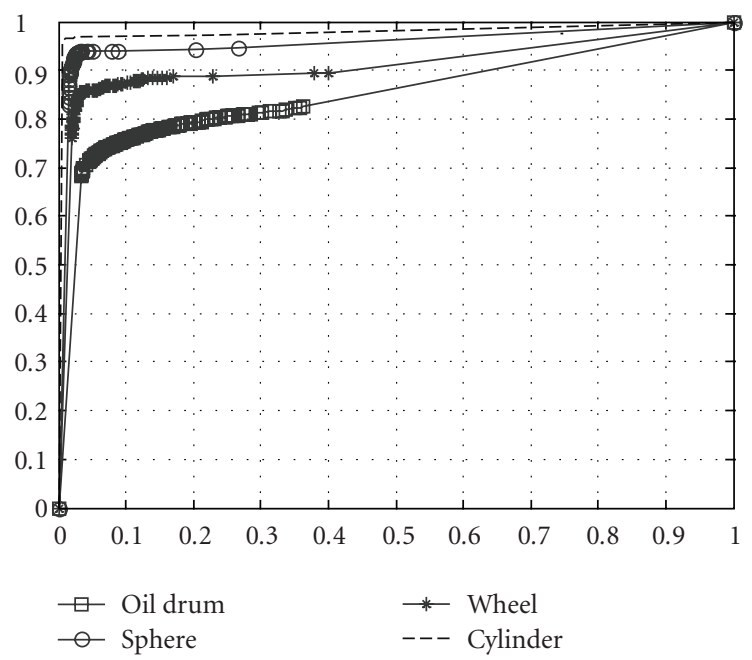

FIGURE 8: ROC curves for four different target classes. X-axis represents the false alarm rate (non targets classified as targets) and the Y-axis is the success rate (targets classified as targets).

Of particular focus of this article is the sensitivity of the classification system to variations on the parameters describing the sonar acquisition geometry.

Aspect angle, for instance, was found to be a critical characteristic for identifying oil drums, but relatively noninformative for cylinders. End-fire aspects (aspect angle from 75 to 125 degrees) make oil drums resemble other classes of similar dimensions, but end-fire cylinders are still well discriminated (Figure 9). The most discriminative features in this case belong to the low backscatter area of the objects, which are sufficient to estimate the sizes of the cylinders. The low performance observed for the end-fire oil drums lowers the overall classification performance of the proposed system for that class (Figure 8).

Grazing angle has also a strong impact in the viewing geometry. It determines the amount of energy that will be returned to the sensor via different paths. Higher grazing angles produce more reverberation from the seafloor and therefore less signal-to-noise ratio (SNR) between the highlight and the background noise coming from the seafloor. The proposed detection and segmentation algorithm assumes that the target has a stronger backscatter signal than the seafloor, which doesn't hold for high grazing angles. The loss in classification performance observed for high grazing angles is mainly caused by that poor detection performance. The poor highlight features are accompanied by shortened shadows, which make the classification even more difficult in these cases.

The performance of the system has also been evaluated for the case where only highlight features are considered (Figure 11). The results obtained are comparable to the ones for the full set of geometrical features (Figure 8), which means that a good part of the discriminative information are located in the highlight segment. That information can only be exploited if the imaging sensor has enough resolution to capture the details in the echo structure, something that

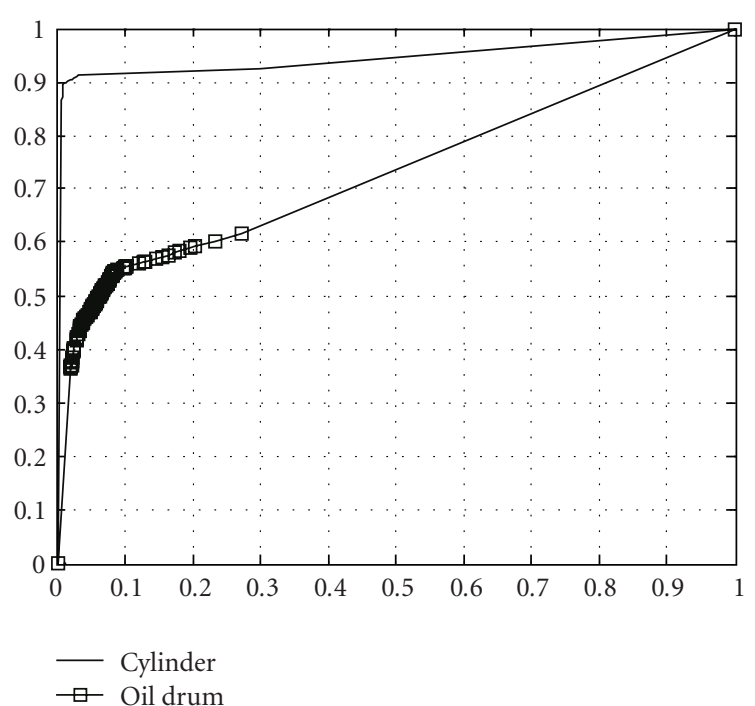

FIGURE 9: ROC curves for the man-made target classes without rotation symmetry (oil drum, cylinder) when the objects are observed end-fire (aspect angle of the target between 75 and 125 degrees). $\mathrm{X}$-axis is false alarm rate and $\mathrm{Y}$-axis success rate.

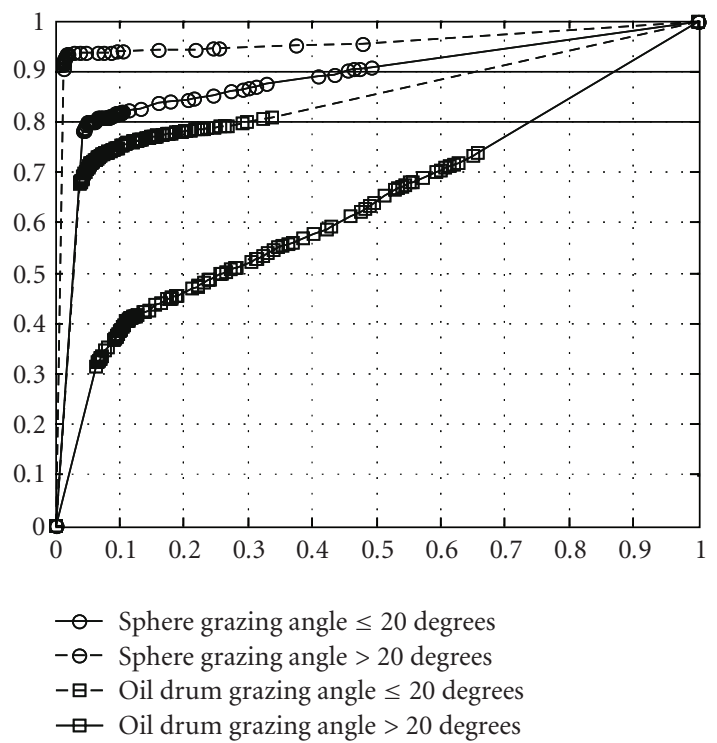

FIGURE 10: ROC curves depending on grazing angle. Grazing angles higher than 20 degrees degrade the system performance significantly. $\mathrm{X}$-axis is false alarm rate and $\mathrm{Y}$-axis success rate.

is only possible for wide ranges when using a modern SAS system.

\section{Results for Real Data}

The limited amount of high-resolution SAS imagery prevented the ATR being tested with real data. However, tests done on the few existing samples available to us have shown promising results, and an extensive set of real images will be available shortly. Training and testing on synthetic dataand validation on the limited available real samples-have 


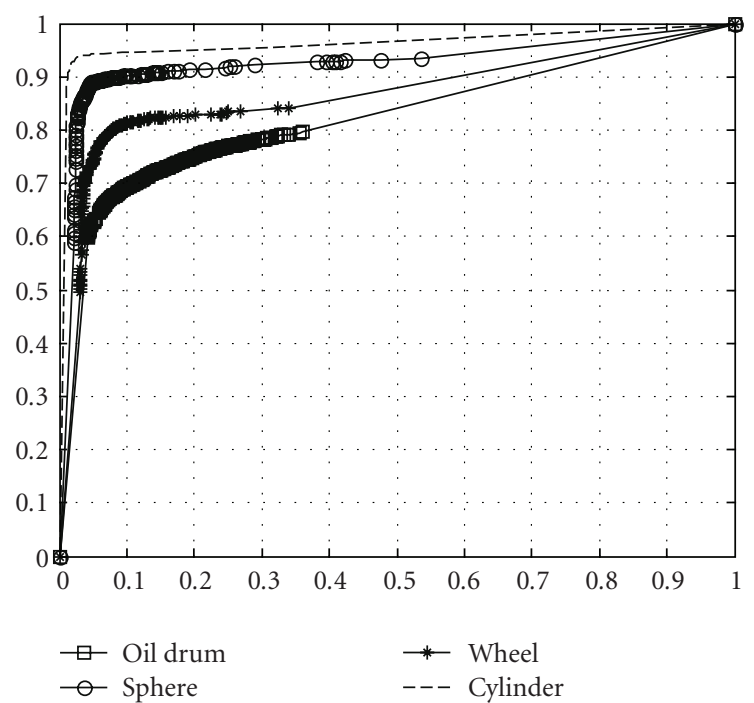

FIGURE 11: ROC curves only considering highlight features, the performance is the same with respect to considering all the features. $\mathrm{X}$-axis represents the false alarm rate (non targets classified as targets) and the Y-axis is the success rate (targets classified as targets).

permitted the system to be fully prepared for testing with real data as soon as a suitable test set is available.

An example of the extracted $C_{h}$ and $C_{s}$ curves for a real image of a cylinder is presented in Figure 12. Comparison of the geometrical features extracted from the image against a training set obtained from the synthetic sample database successfully classify the observed object as a cylinder.

\section{Conclusions}

A system for the extraction of geometrical features for ATR applications has been presented in this paper. The system is composed of two main blocks: segmentation based on the Hilbert transform (HT) and classification based on geometrical features extracted from the segmented image. The system has been trained and tested on a synthetic dataset containing 1528 images has been produced using the SIGMAS model, containing objects of eight different types lying on a flat sea bottom. Different bottom types ranging from soft mud to gravel have been considered (with different scattering strengths), as well as different acquisition geometries (variations in range, grazing angle and aspect angle).

In total 24 geometrical features were computed for each database object. The features were extracted from the highlight, the shadow, and the low backscatter segments of the target image. The best classification results were observed for cylindrical targets, while oil drums proved to be the most difficult to identify for various aspect and grazing angles.

Sensitivity of the classification system to variations in the image acquisition geometry has been studied, with the most influential parameter found to be the grazing angle from the sonar to the target. High grazing angles make the detection

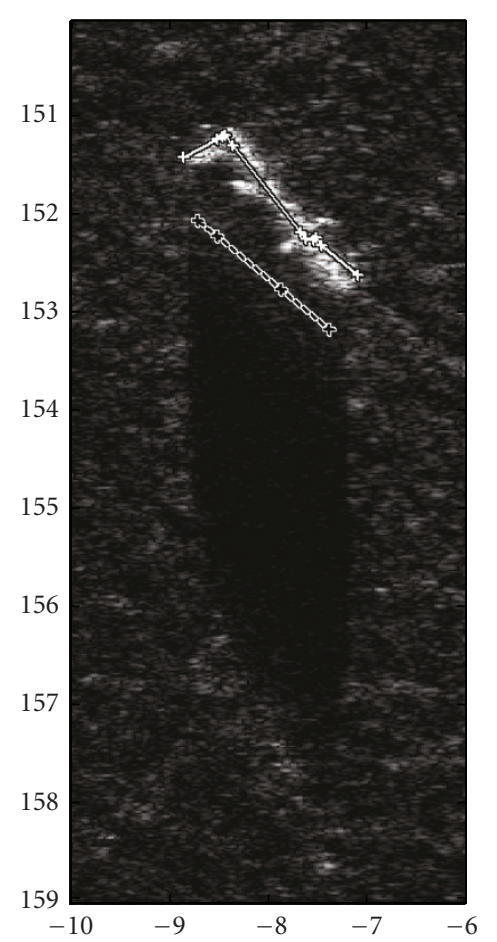

FIGURE 12: In white, the curve $C_{h}(a)$ for the highlight segment from a real image of a cylinder. In black, the curve $C_{s}(a)$ that delimits the start of the shadow region.

task more difficult and therefore lower the performance of the classification stage. Grazing angles below 20 degrees seem to provide the best classification results.

To demonstrate the powerful imaging capabilities obtainable by new SAS sensors, the classification has been performed using only features extracted from the highlight segments. The satisfactory results obtained showed only a slight decrease in performance when compared to the classification using all available features. This shows that the increased resolution of the new SAS sensors is a definitive advantage compared to older underwater imaging systems, primarily utilizing the highly discriminative information that is contained in the details of the target's echo.

Satisfactory results for the limited real data available have been presented. More extensive real datasets are nevertheless required to properly assess the actual performance of the techniques proposed in this paper.

\section{References}

[1] P. Chapman, D. Wills, G. Brookes, and P. Stevens, "Visualizing underwater environments using multifrequency sonar," IEEE Computer Graphics and Applications, vol. 19, no. 5, pp. 61-65, 1999.

[2] P. Blondel and B. J. Murton, Handbook of Seafloor Sonar Imagery, Wiley-Praxis Series in Remote Sensing, John Wiley \& Sons, New York, NY, USA, 1997.

[3] M. P. Hayes and P. T. Gough, "Broad-band synthetic aperture sonar," IEEE Journal of Oceanic Engineering, vol. 17, no. 1, pp. 80-94, 1992. 
[4] J. C. Curlander and R. N. McDonough, Synthetic Aperture Radar: Systems and Signal Processing, Wiley Series in Remote Sensing, John Wiley \& Sons, New York, NY, USA, 1990.

[5] P.-Y. Mignotte, E. Coiras, H. Rohou, Y. Pétillot, J. Bell, and K. Lebart, "Adaptive fusion framework based on augmented reality training," IET Radar, Sonar \& Navigation, vol. 2, no. 2, pp. 146-154, 2008.

[6] S. S. Abeysekera, P. S. Naidu, Y.-H. Leung, and H. Lew, "An underwater target classification scheme based on the acoustic backscatter form function," in Proceedings of the IEEE International Conference on Acoustics, Speech and Signal Processing (ICASSP '98), vol. 4, pp. 2513-2516, Seattle, Wash, USA, May 1998.

[7] S. Reed, Y. Petillot, and J. Bell, "An automatic approach to the detection and extraction of mine features in sidescan sonar," IEEE Journal of Oceanic Engineering, vol. 28, no. 1, pp. 90-105, 2003.

[8] S. Hahn, Hilbert Transforms in Signal Processing, Artech House, Boston, Mass, USA, 1996.

[9] E. Coiras and J. Groen, "Simulation and 3D reconstruction of side-looking sonar images," in Advances in Sonar Technology, V. Kordic, Ed., chapter 1, IN-TECH, Vienna, Austria, 2009.

[10] "APL-UW high-frequency ocean environmental acoustic models handbook," Tech. Rep. 9407, Applied Physics Laboratory, University of Washington, Seattle, Wash, USA, October 1994.

[11] G. M. Livadas and A. G. Constantinides, "Image edge detection and segmentation based on the Hilbert transform," in Proceedings of the IEEE International Conference on Acoustics, Speech and Signal Processing (ICASSP '88), vol. 2, pp. 11521155, New York, NY, USA, April 1988.

[12] S. Venkatesh and R. A. Owens, "On the classification of image features," Pattern Recognition Letters, vol. 11, no. 5, pp. 339349, 1990.

[13] A. Rosenfeld, "Introduction to machine vision," IEEE Control Systems Magazine, vol. 5, no. 3, pp. 14-17, 1985.

[14] R. M. Haralick and L. Shapiro, Computer and Robot Vision, Addison-Wesley, Reading, Mass, USA, 1992.

[15] J. Shlens, "A tutorial on principal component analysis," School of Computer Sciences, Carnegie Mellon University, 2005, http://www.snl.salk.edu/ shlens/pub/notes/pca.pdf.

[16] L. Breiman, J. Friedman, C. J. Stone, and R. A. Olshen, Classification and Regression Trees, Chapman \& Hall/CRC, Boca Raton, Calif, USA, 1993.

[17] M. J. Procopio, T. Strohmann, A. R. Bates, G. Grudic, and J. Mulligan, "Using binary classifiers to augment stereo vision for enhanced autonomous robot navigation," Tech. Rep. CUCS-1027-07, University of Colorado, Boulder, Colo, USA, April 2007.

[18] T. Fawcett, "ROC graphs: notes and practical considerations for researchers," HP Laboratories, Palo Alto, Calif, USA, March 2004. 